\title{
Heartbeat: improving outcomes after myocardial infarction
}

doi:10.1136/heartjnl-2018-314028

Primary percutaneous coronary intervention for acute ST elevation myocardial infarction (STEMI) dramatically improves clinical outcomes compared with unreperfused STEMI. However, acute restoration of myocardial blood flow to infarcted tissue may cause further damage, with reperfusion injury accounting for up to $50 \%$ of infarct size. To date, therapeutic interventions to reduce reperfusion injury that appeared promising in experimental models have not been shown to improve clinical outcomes in patients. In this issue of Heart, Engstrøm and colleagues report the extent of myocardial salvage, as assessed by MRI, in 243 patients with STEMI randomised to danegaptide versus placebo. ${ }^{1}$ Danegaptide in a dipeptide that interacts with connexin-43, resulting in increased gap junction conductance which has been shown to reduce infarct size in animal reperfusion studies. Unfortunately, the myocardial salvage index was no different in patients receiving two different does of danegaptide compared with the control group and there was no difference in clinical outcomes (figure 1).

Is reperfusion injury inevitable or would other therapeutic targets be effective? In an editorial, Johnstone and Isakson ${ }^{2}$ point out some of the challenges in pharmacological approaches to preventing reperfusion injury including delivery of the agent to tissue distal to the coronary occlusion, timing of delivery with the agent only reaching the tissue after reperfusion, failed reperfusion, microvascular obstruction, haemorrhage and oxidative stress. In addition, 'Time to damage and the window for repair is also a critical consideration in therapeutically targeting ischaemia-reperfusion injuries.' 'However, in the best-case scenario, patients will not receive treatment until at least 1.5-2 hours postischaemia. Therefore, it is not clear if the window of opportunity for treatment is missed. This may be a confounding and insurmountable variable in the treatment of patient with STEMI, regardless of the end target.'

Correspondence to Professor Catherine M Otto, Division of Cardiology, University of Washington, Seattle,WA 98195, USA; cmotto@uw.edu

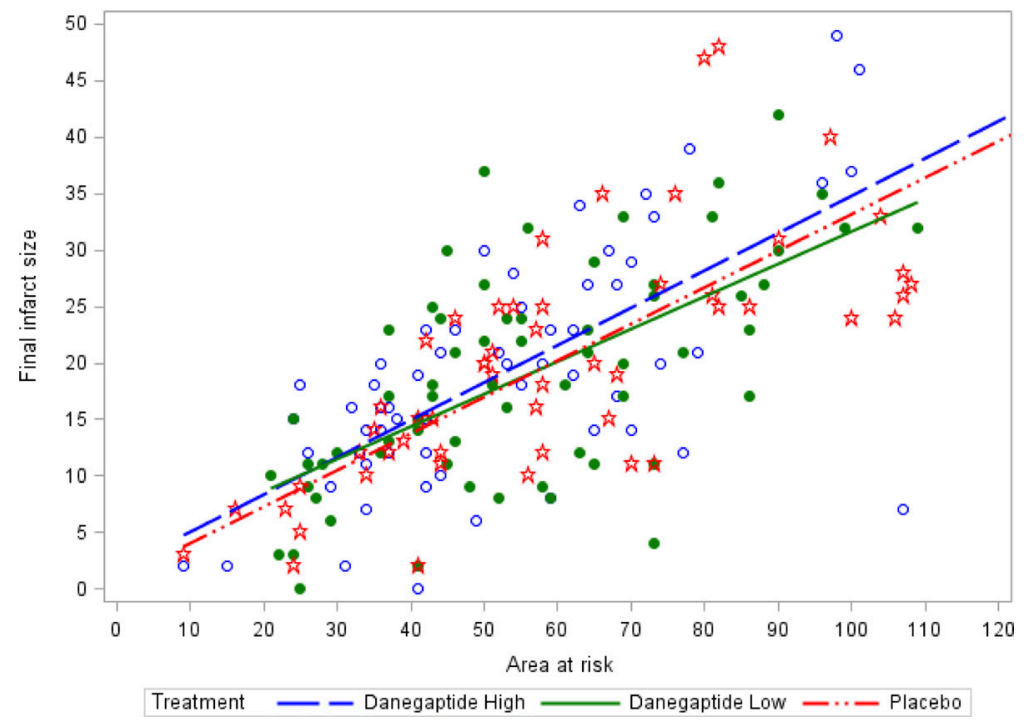

Figure 1 Final infarct size as a function of AAR. Infarct size correlated well with AAR for all treatment groups (control: $r^{2}=0.753, p<0.0001$, danegaptide high: $r^{2}=0.496, p<0.0001$, danegaptide low: $\left.\mathrm{r}^{2}=0.407, \mathrm{p}<0.0001\right)$. AAR, area at risk.

Two other papers in this issue focus on optimal antiplatelet therapy after an acute coronary syndrome (ACS). Current guidelines recommend dual antiplatelet therapy (DAPT) with aspirin (a cyclooxygenase inhibitor) plus a $\mathrm{P}_{2} \mathrm{Y}_{12}$ inhibitor. Randomised clinical trials (RCTs) have shown that the newer P2Y $\mathrm{Y}_{12}$ inhibitors are superior to clopidogrel for reducing major cardiovascular events, although with an increased risk of bleeding, but the potential benefit in an unselected patient population remains controversial because RCT patient selection may not represent the full clinical spectrum of patients with ACS. In this issue of Heart, Edfors and colleagues ${ }^{3}$ address this concern using data from a large Swedish registry of over 45000 patients with myocardial infarction. In this large 'real-life' population, those discharged on ticagrelor had a lower 1-year risk of the composite outcome (death, myocardial infarction and stroke) and a higher risk of bleeding compared with those discharged on clopidogrel, even across subgroups defined by estimated glomerular filtration rate (figure 2).

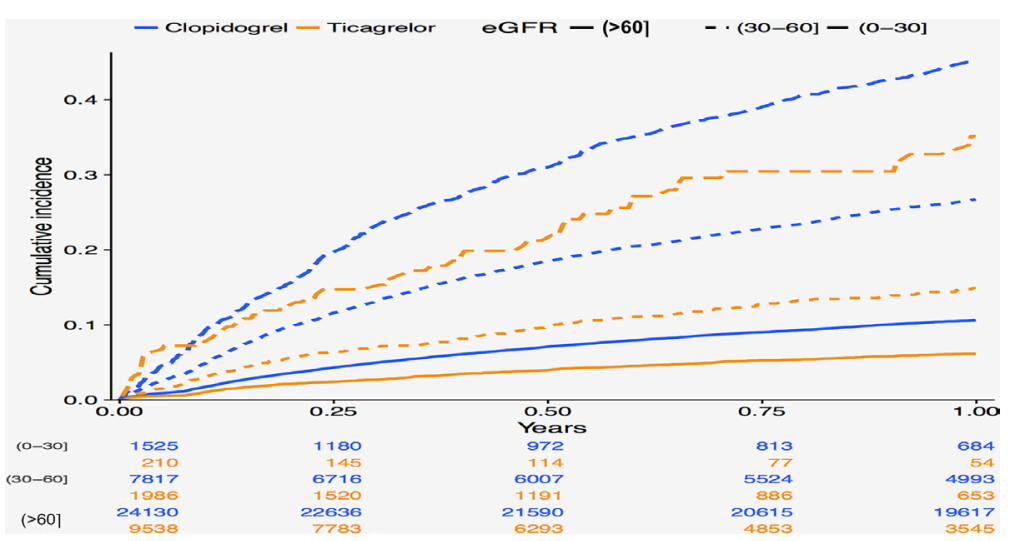

Figure 2 Cumulative incidence rates for the combined outcomes (death, myocardial infarction or stroke) and numbers at risk stratified on estimated glomerular filtration rate (eGFR) groups in $\mathrm{mL} /$ $\min / 1.73 \mathrm{~m}^{2}$. 


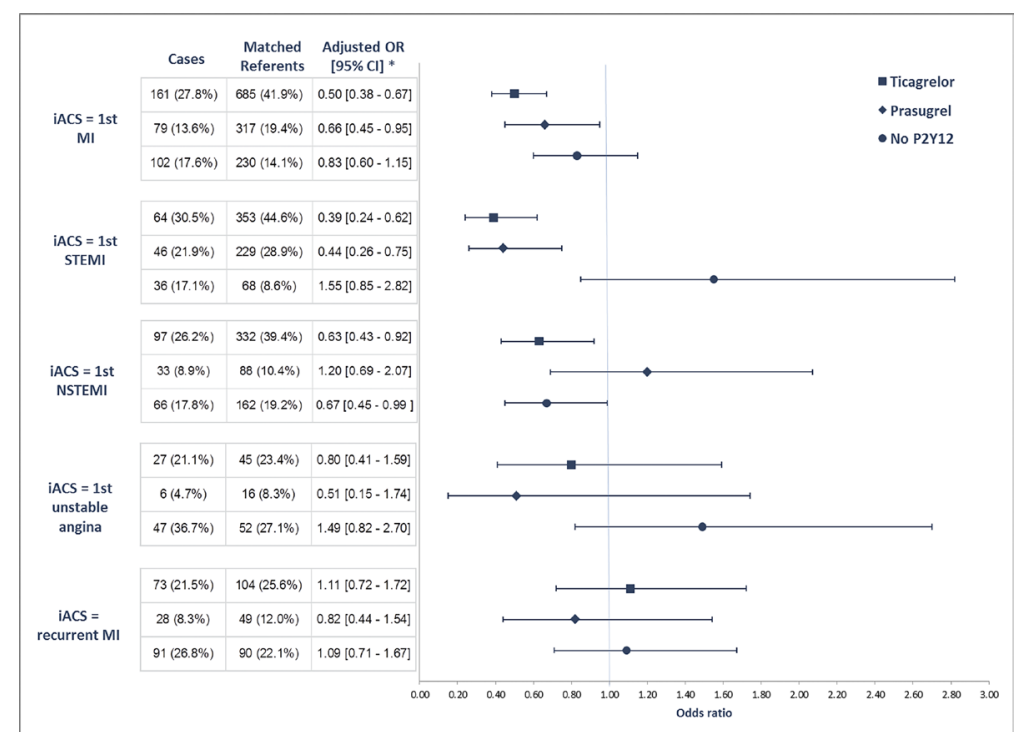

Figure 3 Association between reMI and ITT exposure to APAs by index ACS. ACS, acute coronary syndrome; APA, antiplatelet agent; iACS, index ACS; ITT, intention-to-treat; MI, myocardial infarction; reMI, recurrent MI; STEMI, ST- elevation MI, NSTEMI, non-STEMI.

A second study examined the effectiveness of ticagrelor or prasugrel for prevention of recurrent myocardial infarction using a case-referent study design ${ }^{4}$. In these 1047 cases and 2234 matched controls, treatment with either ticagrelor or prasugrel was associated with lower risk of recurrent myocardial infarction compared with treatment with clopidogrel. ${ }^{4}$ Again, these findings were consistent across subgroups including patients with their first myocardial infraction, STEMI and non-STEMI patients and those who were 70 years of age or older (figure 3).

In an editorial, Onwordi and colleagues ${ }^{5}$ conclude 'Optimal antithrombotic therapy in ACS remains a subject of debate and the balance between ischaemic risk reduction and bleeding is often fine. In this edition of Heart, evaluation of registry data sheds further light on the effects of DAPT in the real world and compares favourably with the outcomes seen in RCTs. This may alleviate clinician concerns and support further uptake of guideline-recommended therapy. Still, little is known about the clinical effects of ticagrelor in dialysis-dependent patients and the very elderly, so more research is needed in these groups.'

Other interesting papers in this issue include a comparison of general versus non-general anaesthesia in patients undergoing transfemoral transcatheter aortic valve implantation (TAVI). Compared with non-general anaesthesia, general anaesthesia was associated with a longer hospital length of stay and longer procedural duration with no difference in procedure outcomes, and 30-day and 1-year mortality. ${ }^{6}$ Based on data like this, most valve centres now no longer use general anaesthesia for patients undergoing transfemoral TAVI, unless there are other reasons for considering this approach in an individual patient.

Nutritional factors related to risk of cardiovascular disease continue to be of great interest. In a large epidemiological study, Larsson and colleagues found that eating nuts at least three times a week was associated with an $18 \%$ lower risk of atrial fibrillation, as well as a reduced risk of heart failure. ${ }^{7}$ Nut consumption was not associated with risk of fatal myocardial infarction, aortic valve stenosis or stroke.

The use of meta-analysis to inform evidence-based medicine guideline recommendations is not yet accepted by all clinicians. In a provocative editorial entitled 'Meta-analysis: mistake or milestone in medicine', Foroutan and colleagues ${ }^{8}$ argue that 'systematic reviews and meta-analyses are powerful and informative. They should not be condemned as a form of medical fake news. There will always be poorly conducted randomised trials of little use (and potentially misleading)-the same is true of meta-analysis. When faced with a meta-analysis, clinicians should look at its methods and results critically—and use the guidance available to help them in doing so. There is no doubt, however, that optimal clinical care requires knowledge of the best available evidence, and systematic summaries are required to be confident that clinicians indeed have access to that evidence. With rigorous systematic summaries, we can be confident as we partner with patients to ensure that they achieve the best possible outcomes.'

The Education in Heart article in this issue $^{9}$ discusses the use of transcatheter valves for treatment of congenital heart disease, particularly in the pulmonic position. The use of multimodality imaging is illustrated, along with the rapidly evolving technology available for transcatheter repair procedures.

The Image Challenge ${ }^{10}$ question asks you to interpret the angiogram in a 42-year-old woman presenting with an anterior STEMI; an interesting finding in the external iliac artery.

Competing interests None declared.

Patient consent Not required.

Provenance and peer review Commissioned; internally peer reviewed.

(C) Author(s) (or their employer(s)) 2018. No commercial re-use. See rights and permissions. Published by BMJ.

Check for updates

To cite Otto CM. Heart 2018;104:1553-1554.

Heart 2018;104:1553-1554.

doi:10.1136/heartjnl-2018-314028

\section{REFERENCES}

1 Engstrøm T, Nepper-Christensen L, Helqvist S, et al. Danegaptide for primary percutaneous coronary intervention in acute myocardial infarction patients: a phase 2 randomised clinical trial. Heart 2018:104:1593-9.

2 Johnstone SR, Isakson BE. 'Gaps' in targeted ischaemic injury therapies in ST-elevation myocardial infarction. Heart 2018;104:1557-8.

3 Edfors R, Sahlén A, Szummer K, et al. Outcomes in patients treated with ticagrelor versus clopidogrel after acute myocardial infarction stratified by renal function. Heart 2018:104:1575-82.

4 Grimaldi-Bensouda L, Danchin N, Dallongeville $J$, et al. Effectiveness of new antiplatelets in the prevention of recurrent myocardial infarction. Heart 2018;104:1583-92.

5 Onwordi ENC, Parker WAE, Storey RF. Dual antiplatelet therapy in the 'real world'. Heart 2018:104:1555-6.

6 Eskandari M, Aldalati O, Dworakowski R, et al. UK TAVI Steering Committee and the National Institute for Cardiovascular Outcome Research. Comparison of general anaesthesia and non-general anaesthesia approach in transfemoral transcatheter aortic valve implantation. Heart 2018;104:1621-8.

7 Larsson SC, Drca N, Björck M, et al. Nut consumption and incidence of seven cardiovascular diseases. Heart 2018:104:1615-20.

8 Foroutan F, Guyatt G, Alba AC, et al. Metaanalysis: mistake or milestone in medicine? Heart 2018; 104:1559-61.

9 Ghobrial J, Aboulhosn J. Transcatheter valve replacement in congenital heart disease: the present and the future. Heart 2018;104:1629-36.

10 Fahey JK, Ihdayhid AR, White AJ. A 42-year-old woman with acute myocardial infarction. Heart 2018;104:1628. 